\title{
Application of a location model to fire stations in Rotterdam
}

\author{
J.A.M. SCHREUDER \\ Department of Applied Mathematics, Twente University of \\ Technology, Enschede, The Netherlands
}

Received November 1979

Revised January 1980

This report is an outgrowth of a research project carried out by the Operations Research Group of Twente University of Technology in cooperation with the Department liire-Brigade Affairs (Home Office) and the Rotterdam Fire Department. The objectives of the project were to determine the minimal number of fire stations, their locations and the number of first attendance pumpers, so that each point in town can be reached within a prescribed attendance time with sufficient equipment.

A road network approach was used for determining the set of possible location areas for fire stations followed by a set-covering approach for calculating the minimal number of stations. The hard points to tackle were the construction of robust networks, the determination of the possible location areas and the calculation of all the alternative locations of a minimal number of fire stations.

A simulation was carried out to test whether the solutions given by the network approach were realistic.

Keywords: Fire station, location, network, set-covering, simulation

\section{Problem}

Upto 1972, the Rotterdam Fire Department was part of 'ROTEB', which was a public service not only dealing with fire protection, but also, among other things, with garbage collection and transportation services. As a result of this organizational set-up, the location of the ROTEB-stations (delivering combined services), was not entirely satisfactory for providing optimal fire protection services. Some stations were located in the neighbourhood of rivers or canals (e.g. the river 'Nieuwe Maas') or railways and could only attend calls within the prescribed attendarice time in half of the area that could be reached if the stations

(c) North-Holland Publishing Company

European Jourinal of Operational Research 6 (1981) 212-219 were more centrally located in the given district; see Appendix and Vossenaar [13].

When, in 1972, the Rotterdam Fire Department became mostly professional and independent, the City Counsil decided to investigate a possible reorganization of the Fire Department. For fixing the location, the minimal number and the capacity of the fire stations, the planning commission decided to make use of OR-methods and contacted the 'Emergency Transportation Group' of the Twenty University of Technology.

The objectives of the resulting project were to determine

- the minimal number of stations in the Northern and the Sourthern part of Rotterdam (naturally divided by the main river the 'Nieuwe Maas');

- the location of these stations;

- the number of first attendance pumpers at each station;

- the robustness of the proposed solution.

\section{Location model}

\subsection{Assumptions}

In The Netherlands fire cover is planned in reference to risk classification. Each risk category has standards for first attendance and first attendance times, see Table 1. These standards give the norms for the attendance time of the first attendance cars: TAS (Tank$A$ uto-Spuit = low pressure pumper).

Attendance time is turn-out time + travel time. Turn out time is the time between the receipt of a fire call and the departure of the first pumper from the station. The average turn out time for the professionals is one minute ard for volunteers three minutes. In this study one minute is used. Travel time is the time from the station to the fire-address.

The standard attendance times for other cars (i.e., ladder trucks) and 'technical assistance' are much wider and unlike, e.g., in the U.S.A., not considered as determining factors for the location of the stations. Also the double covering principle was used. This means that all points in $\mathrm{A}, \mathrm{B}$ and $\mathrm{C}$ risk districts should be reachable from two different stations within 
Table 1

Norms for first attendance service

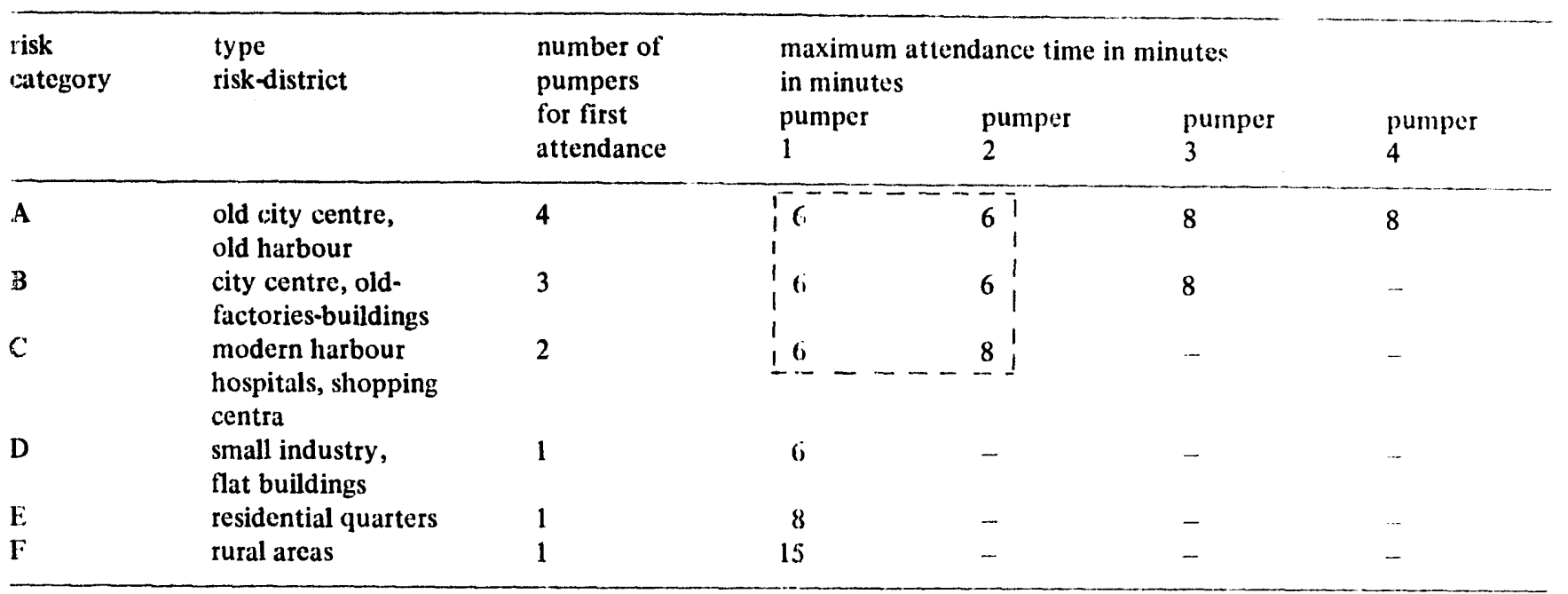

the appropriate standard times, see the dashed line in Table 1, this to decrease the chance for delay as a result of traffic congestion.

Historical data pointed out that the pumpers could hold an average speed of $40 \mathrm{~km} / \mathrm{h}$. Of course, this does not hold for the congestion hours on some roads, but this can be tackled as described in the conclusion. Because of the increasing traffic during the last years and to test the sensitivity of the obtained solutions, we also took an average speed of $36 \mathrm{~km} / \mathrm{h}$.

Because most of the day the tunnels and bridges connecting North and South are blocked by the traffic, it was decided to look at the locations in North and South as different parts.

Since a number of stations were recently built and therefore could not be replaced, the model incorporated from the outset certain locations as already fixed: three in the Northern part (1.1, 1.2 and 1.4) and four in the Southern part $(2.1,2.3,3.4$ and 2.6), see Appendix 1.

We used the present roadmap of the municipality of Rotterdam and the geographical area as presented in Appendix 1.

\subsection{Network}

We used a network approach for finding the set of possible location areas for fire stations, i.e., those location areas on the network which give a maximal service time, as predetermined by the given norms in Table 1.

For the area covered by the Rotterdam Fire Depart- ment, a network representation was constructed. The edge-set was formed by roads effectively used by the pumpers, the node-set, in turn, was determined by the roadcrossings.

For this, the area was divided in a number of districts. These districts were designed in such a way that their borders coincide, as much as possible, with obstacles such as rivers, railways and parks. Also it is desirable that each district has one risk classification. The nodes (roadcrossings) were determined in such a way that in each district there was one node and that node was, as much as possible, located in the centre of that district.

If there is a fire call in a district we assumed the following, see Fig. 1.

The pumper of the nearest fire station will turn-out . It will use the roads of the network to the node that represents the district centre and from there to the fire address.

The travel times between the nodes are found by dividing the measured distance on a roadmap through the average speed. Also it is clear that we do not really need the same average speed for the whole city. We can establish different speeds between each pair of nodes if needed. In fact we gave a few roads (e.g., express roads) a higher average speed.

In each district we determined a socalled most difficult point, which has the longest travel time with respect to the district centre. Note, the main restriction imposed on our analysis was, that every point in town has to be reachable within the prescribed norms, cf. Table 1 . In this way we knew the maximal district 


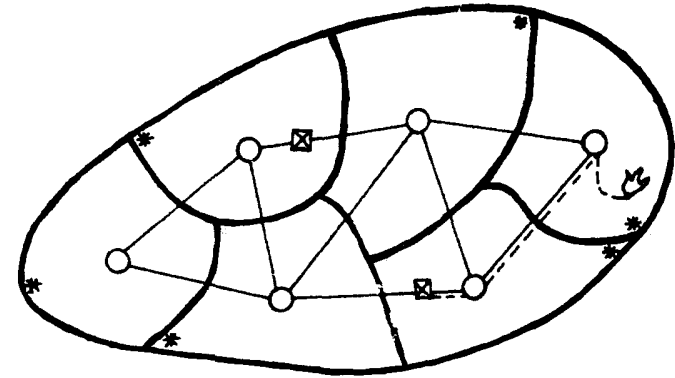

Fig. 1. Example network.

time, i.e., the time needed to travel from the district centre to the most difficult point.

Next we determined for each district the corrected time, i.e., the attendance time according to the norm for that district minus the maximal district time minus the one minute turn-out-time.

With the corrected time we calculated the 'reach' of each node: how far away can a fire station be located on the network in order that we still can fulfill the attendance requirements. For calculating these 'reaches', we used a shortest route algorithm bases on the method of Dijkstra [4]. Note that the end-points of these 'reaches' are in general not in a node.

To make the network more realistic, extra nodes had to be added in order to describe the actual routes followed by the fire-brigade. These nodes represent no district centre and no 'reach' had to be calculated. Special attention had to be given here to the double covering and different attendance times, resulting in two different 'reaches' for these nodes. We choosed the network in such a way that all existing and planned fire stations were on the network. A station can be fixed by giving its node a zero 'reach'.

By intersecting of all 'reaches' we found all the possible location areas. Each area so obtained covers a set of nodes within the norms. We looked for only those areas whose set of nodes is not a subset of any other area.

Detailed information about the networks and finding the possible location areas can be found in van Loon and Schreuder $[9,10]$.

\subsection{Set-covering}

We have to select from all the possible location areas that minimal number (even all the alternatives) which still fulfils the first attendance norms.

We can formulate this problem as an integer programming problem. Let $I_{1}$ be the set of districts which node

fire station

fire address

most difficult point

district border

road

route used by pumper must be covered, $I_{2}$ the districts where double coverage is necessary, $a_{i j}=1$ if district $i$ is reachable from possible location area $j$ and $a_{i j}=0$ otherwise, $n$ the number of possible location areas (up to 50), $x_{i j}$ a zero-one variable with the value 1 if a fire station is placed on possible location area $j$, than the formulation is

$$
\begin{array}{ll}
\text { minimize } & \sum_{j=1}^{n} 1 \cdot x_{j}, \\
\text { subject to } & \sum_{j=1}^{n} a_{i j} x_{j} \geqslant 1 \quad \text { for } i \in I_{1}, \\
& \sum_{j=1}^{n} a_{i j} x_{j} \geqslant 2 \quad \text { for } i \in I_{2}, \\
& x_{j} \in\{0,1\} \quad \text { for } j=1,2, \ldots, n .
\end{array}
$$

This is known as the set-covering problem, Salkin [12].

Reduction rules are used for reducing the number of possible location areas, see $[10]$. Whether there are possible location areas which have to be always in the solution or never, can be determined with Lagrange Relaxation, see Camerini et al. [3].

We solved the set-covering problem by transforming it to a satisfiability problem - Aho et al. [1] -, see Example 1.

Example 1. A feasible solution (see Table 2) must give the value 1 to the Boolean product

$\left(x_{1} \cdot x_{2}+x_{1} \cdot x_{4}+x_{2} \cdot x_{4}\right) \cdot\left(x_{2}+x_{3}\right)$

where ' + ' Boolean sum and '. ' Boolean product. Meaning that we can place fire stations in possible location areas 1 and 2 or place fire stations in areas 1 and 4 etc. We were only interested in those combinations which gave a minimal number of fire stations. After using the common reduction rules and solution 
Table 2

$a_{i j}$ Matrix

\begin{tabular}{lllll}
\hline node $i$ & \multicolumn{4}{l}{ possible location area $j$} \\
\cline { 2 - 5 } & 1 & 2 & 3 & 4 \\
\hline 1 & 1 & 1 & 0 & $1(\geqslant 2)$ \\
2 & 0 & 1 & 1 & $0(\geqslant 2)$ \\
\hline
\end{tabular}

method for Boolean problems, see Hammer and Rudeanu [6], there are two alternative solutions: place fire stations in possible location areas 1 and 2 or 2 and 4.

Selecting from the alternative slutions those ones with a minimal total number of first attendance pumpers, can be done with nearly the same Boolean method.

After placing one pumper in each station, we can reduce the remaining problem - we have still to fulfil the norms with regard to the third and fourth pumper, see Table 1 - to again a set-covering problem.

Instead of placing one station in one possible location area, we can put now (if required) one or two ixtra pumpers in one station.

In Example 1 the Boolean product would change as follows:

$\left(x_{1} \cdot x_{1}^{\prime}+x_{1} \cdot x_{2}+x_{1} \cdot x_{4}+x_{2} \cdot x_{2}{ }^{\prime}+x_{2} \cdot x_{4}\right.$

$\left.+x_{4} \cdot x_{4}^{\prime}\right) \cdot\left(x_{2}+x_{3}\right)$.

Meaning that we can put two pumpers in fire station 1 or one pumper in station 1 and one pumper in station 2 , etc.

\subsection{Difficulties in application}

An important question in solving a location model is to determine a set of possible location areas for the fire stations, e.g., see Plane and Hendrick [11]. Unfortunately in The Netherlands there is no correlation between the response times and the response distances calculated from the coordinates of the fire stations and the incidents. The main reason is that the cities in The Netherlands have, unlike in the U.S.A., no rectangular streetsystem.

In contrast to the study of Plane and Hendrick, it was easy to obtain absolute time requirements; they were already determined by the Fire Department, see norms of Table 1.
In view of these facts, we choose a network approach, see van Loon [8].

If possible location areas are restricted to the nodes, the problem is called a $P$-centre problem, whereas, if they are allowed on the whole network, it is called an absolute P-centre problem. In our case, finding just the $P$-centre made no sense, because for sufficient accuracy we need so rnuch extra nodes, that the solutions converge to the solutions of the absolute $P$-centre. Our algorithm is based on that of Christofides and Viola [2].

Because the fire-brigade has more tasks (and equipment) than the first attendance service, the manning (and costs) of the stations could not be considered by our model. Furthermore, this made it impossible to compare the costs of stations and the pumpers. The consequence was that the location of the stations and of the pumpers were not calculated together. First we computed the location of the fire stations, selected then from the alternatives those ones with the minimal number of first attendance pumpers.

Establishing the network was the crucial part of the whole investigation. Choosing the network districts, its most difficult points, the roads and the nodes of the network had been done in : iuse cooperation with the Fire Department.

Experienced drivers of the fire-brigade had to be questioned for the actual used routes and quite a lot of experiments had to be made for an acceptable chioce of the districts. Compromises had to be made between the accuracy of the network and the capacity and possibilities of the computer program. To reach an acceptable network - including the possibility for sensitivity analysis - involves a considerable effort.

We choose the Boolean method for solving the setcovering problem, because in our case the reduction rules worked extremely well out and it was easy to obtain all the alternative optimal solutions. Solving the problem was a matter of seconds in computer-time.

\section{Results}

In the approach with no stations ficied and average speed $40 \mathrm{~km} / \mathrm{h}$, the program gave in North six stations with one pumper in each station and in South five stations with one pumper in each station except in station 2.3, where two pumpers were needed.

In the more realistic situation where some stations were fixed, one supplementary station with one pum- 
per was needed in the Northern and one in the Southern area.

With an average speed of $36 \mathrm{~km} / \mathrm{h}$, an additional station with pumper(s) was needed in the Northern and the Southern area.

It appears that in North the stations 1.1, 1.2 and 1.10 were well situated. The location of the stations $1.4,1.5$ and 1.9 were badly choosen. The planned station 1.8 was superfluous. The existing location does not satisfy the norms neither at $40 \mathrm{~km} / \mathrm{h}$, nor at 36 $\mathrm{km} / \mathrm{h}$.

In the Southern area the station 2.4 was completely misplaced and also to a lesser extent the stations 2.2 , 2.5 and 2.6. The planned station 2.3 was well choosen. Like the Northern area, the existing location was not feasible within the norms.

Detailed solutions can be found in $[9,10]$.

\section{Simulation}

Operational aspects of fire stations and pumpers can often been viewed as queuing problems. Questions arise like: What is the probability that no pumper is available when a call comes in? Is the assumption of random arrival of calls valid? Can the time spent in dealing with an incident be approximated by a negative exponential distrihution? Furthermore, we want to test whether the solutions given by the network approach are realistic.

To answer these questions a simulation study was carried out by Gillebaart [5]. The simulation was based upon the daily reports of the fire-brigade of the first half of the year 1975 (South) (he choose new, smaller and three times as much districts as for the networkmodel). From these reports the moments of the call, the fire address, the kind of fire (from fals to disaster), and the magnitude of the fire (number of pumpers needed) were simulated.

Given a certain location of the fire stations, he determined from which station(s) the pumper(s) came (the nearest in distance with a pumper available), the attendance time, the extinction time and the traveltime back to the station. The result was a number of travel times of the first and eventually second pumper in each district. Comparing these times with the norms of each district gave us an indication as to how good or bad certain locations were. The computations of the simulation were based on an average speed of 40 $\mathrm{km} / \mathrm{h}$, this number was based on historical observations.

The simulation showed us that for the location of the fire stations in 1974, especially the second pumper was not within the norms. The planned stations gave an improvement, but were still not sufficient. The locations resulting from our network model were all within the norms.

Another interesting result of the simulation was that generation of the moment of the fire calls cannot be characterized by a stationary Poisson-process. Better results are obtained by a time dependent (time of the day) Poisson-process.

\section{Conclusions}

In the Final Report of the "Commission Reorganization Fire Brigade Aifairs' the sesults of our location model were adopted. This means that in RotterdamNorth 7 and in -South 6 fire stations with one first attendance pumper in each station are needed in the realistic situation.

Decreasing the number of fire stations while maintaining the present state of fire risk covering is only possible if the very high initial expenses (building of a set new stations) are consciously accepted.

In view of the increasing traffic intensities, it looks desirable that the number of stations would increase in the future, see [14].

After the publication of the Final Report the following changes in the location of the fire stations were made. The stations 2.2 and 2.5 are closed down and 2.1,2.3 and 2.7 are open. New stations are planned in 1.7 and 1.10 . The station 1.3 will be closed down before 1982. The location of its replacement is not made public yet, see Appendix 2.

Despite the approximative character of our location model, it proved to be of practical value in generating acceptable solutions within prescribed norms. Even the problem of dealing with the congestion hours (some districts can be completely blocked) becomes tractable with the aid of the model. We can, if wanted, define different average speeds beiween each pair of nodes. In this way the question of locating first attendance pumpers during the congestion hours can be solved - at present this is done by experience through the fire-brigade. Also the consequences of new circling routes of changed traffic through-put can be determined.

The computerprogram is made available to the Home Office, that has given it a trial run in Maastricht.

In close cooperation with the Home Office further research is going on and planned on the following topics: 
- the location of equipment other than the first attendance pumpers;

- the consequences of a higher rate of calls, especially the calls during a given attendance;

- reallocation of the remaining first attendance pumpers in case of a disaster; maximal covering, see e.g., Klastorin [7];

- reallocation of five stations in case of cooperation between two or more municipalities - Fire-brigade affairs are according to the Dutch Law a matter for each municipality with the Major having prime responsbility. The Home Office can only give advice, coordinate, distribute the subsidies, provide for training facilities etc., but has no authority on the firebrigades;

- studying the consequences of other norms.

\section{Appendix 1. Location fire stations in 1974}

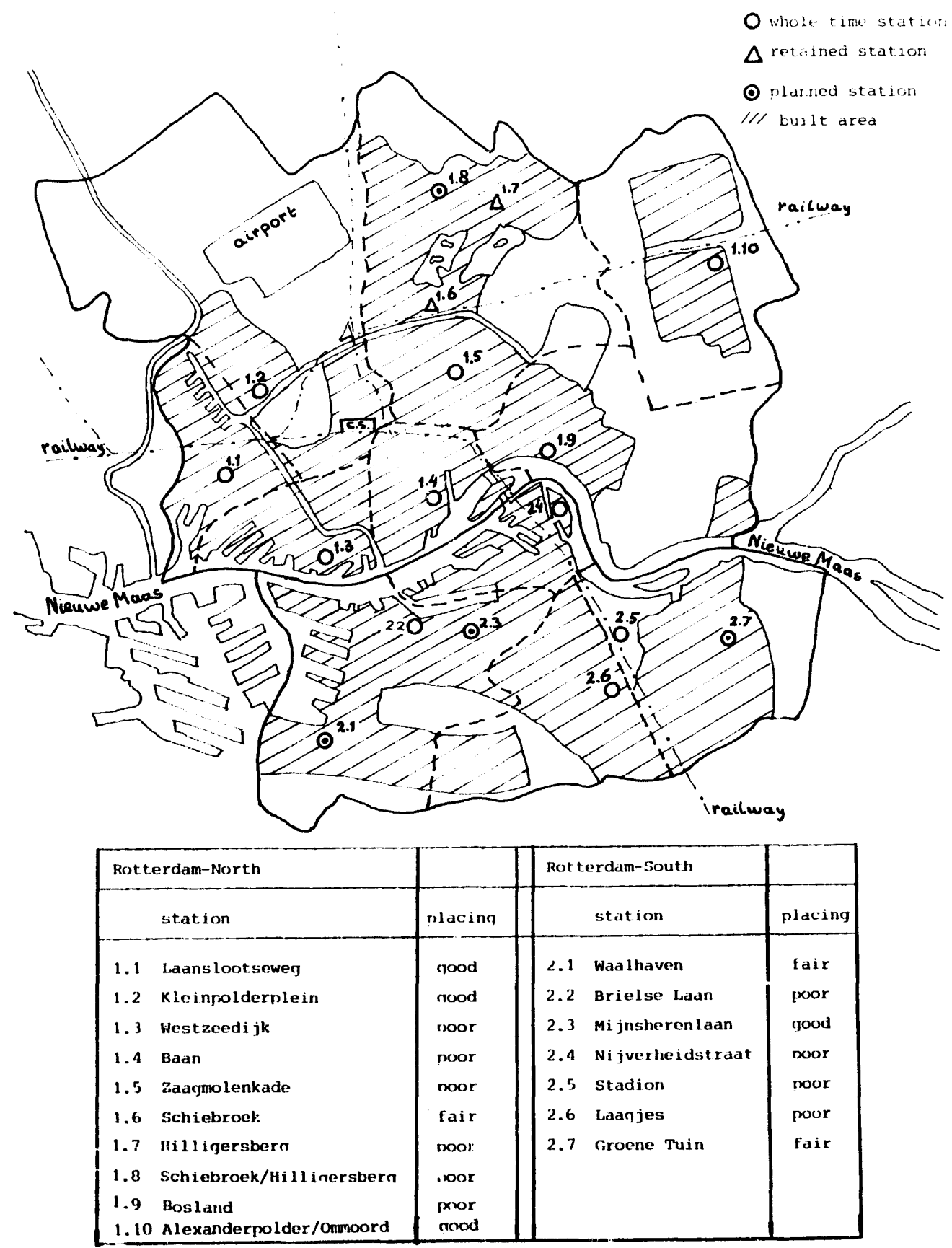




\section{Appendix 2. Location fire stations in 1979}

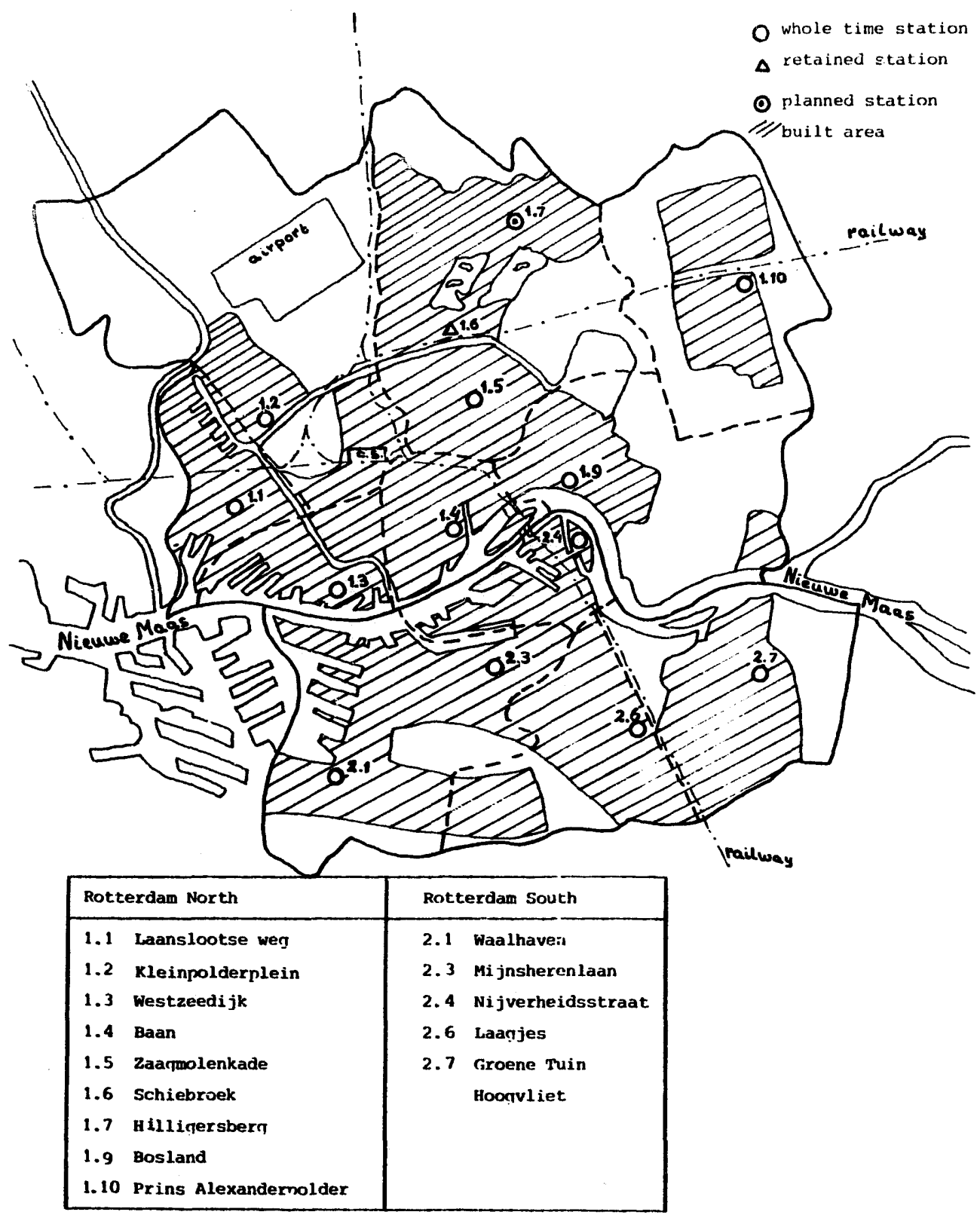

\section{References}

[1] A.V. Aho, J.E. Hopcraft and J.D. Ullman, The Design and Analysis of Computer Algorighms (Addision-Wesley, Reading, MA, 1974).

[2] N. Christofides and P. Viola, The optimal location of multi-centres on a graph, Operational Res. Quart. 22 (1971) 145 .

[3] P.M. Camerini, L. Fratta and F. Mhaffioli, On improving relaxation methods of modified gradient techniques, Math. Programming Study 3 (1975) 26-34.
[4] E. Dijkstra, A note on two problems in connection with graphs, Numer. Math. 1 (1959) 269-271.

[5] A. Gillebaart, Simulation location fire stations (in Dutch), Baccelors Thesis, Twente University of Technology (1977).

[6] P.L. Hammer and S. Rudeanu, Boolean methods in Operations Research (Springer, Berlin, 1968).

[7] T.D. Klastorin, On the maximal covering location problem and the general assignment problem, Management Sci. 25 (1979) 106-112.

[8] J.N.M. van Loon, On the minimal number and location 
of fire stations and fire appliances, Memorandum No. 104, T.H. Twente, Enschede (1975).

[9] J.N.M. van Loon and J.A.M. Schreuder, On the minimal number, the location and the capacity of fire stations in Rotterdam (in Dutch), Report T.H. Twente, Enschede (1976).

[10] J.N.M. van Loon and J.A.M. Schreuder, On the minimal number and location of fire stations and fire appliances in Rotterdam, in: H. Roubens, Ed., Advances in OR (North-Holland, Amsterdam, 1976).

[11] D.R. Plane and T.E. Hendrick, Mathematical programming and the location of fire companies for the Denver Vire Department, Operations Res. 25 (1977) 563-578.
[12] H.M. Salkin, The set covering problem, in: Integer Programming (Addision-Wesley, Reading, MA, 1975) 439-481.

[13] B. Vossenaar, Note on the organization from 1938 until today and the fire-brigade stations now and in the future (in Dutch), Chief fire-brigade Rotterdam (1973).

[14] B. Vossenaar (chairman) and Mr. H.J.P. van Kempen, Final report of the Commission Reorganization FireBrigade Affairs (in Dutch), Fire Department, Municapality Rotterdam (1976). 